\title{
The oral motor capacity and feeding performance of preterm newborns at the time of transition to oral feeding
}

\author{
M.A. Bauer, L.S. Prade, M. Keske-Soares, L.S.B. Haëffner and A.R.M. Weinmann \\ Departamento de Pediatria e Puericultura, Centro de Ciências da Saúde, Universidade Federal de Santa \\ Maria, Santa Maria, RS, Brasil
}

Correspondence to: A.R.M. Weinmann, Departamento de Pediatria e Puericultura, Centro de Ciências da Saúde, Universidade Federal de Santa Maria, Av. Roraima, 1000, 97105-900 Santa Maria, RS, Brasil

Fax: +55-55-3220-8520. E-mail: armweinmann@smail.ufsm.br

\begin{abstract}
The objective of the present study was to determine the oral motor capacity and the feeding performance of preterm newborn infants when they were permitted to start oral feeding. This was an observational and prospective study conducted on 43 preterm newborns admitted to the Neonatal Intensive Care Unit of UFSM, RS, Brazil. Exclusion criteria were the presence of head and neck malformations, genetic disease, neonatal asphyxia, intracranial hemorrhage, and kernicterus. When the infants were permitted to start oral feeding, non-nutritive sucking was evaluated by a speech therapist regarding force (strong vs weak), rhythm (rapid vs slow), presence of adaptive oral reflexes (searching, sucking and swallowing) and coordination between sucking, swallowing and respiration. Feeding performance was evaluated on the basis of competence (defined by rate of milk intake, $\mathrm{mL} / \mathrm{min}$ ) and overall transfer (percent ingested volume/total volume ordered). The speech therapist's evaluation showed that $33 \%$ of the newborns presented weak sucking, $23 \%$ slow rhythm, $30 \%$ absence of at least one adaptive oral reflex, and $14 \%$ with no coordination between sucking, swallowing and respiration. Mean feeding competence was greater in infants with strong sucking fast rhythm. The presence of sucking-swallowing-respiration coordination decreased the days for an overall transfer of $100 \%$. Evaluation by a speech therapist proved to be a useful tool for the safe indication of the beginning of oral feeding for premature infants.
\end{abstract}

Key words: Preterm; Oral motor conditions; Feeding performance

Received November 8, 2007. Accepted October 21, 2008

\section{Introduction}

Advances in medical science are responsible for the improvement observed in intensive neonatal care, permitting the survival of infants with various conditions and of different gestational ages, including those born at the limit of viability. These results, however, are frequently obtained after long periods of hospitalization and prolonged mechanical ventilation which, added to neurological, gastrointestinal and cardiorespiratory immaturity, may account for the feeding difficulties occurring in this group of infants.

In newborns before 34 weeks of gestational age it is necessary to use a feeding tube, which deprives the newborn of sensory stimuli, impairing its motor-oral development, possibly altering the sucking-swallowing-respiration coordination and thus impairing oral feeding even more (1$3)$. A delay in the acquisition of oral feeding implies a poorer feeding performance, which in turn may influence the duration of hospitalization in the neonatal unit.

The postmenstrual age (PMA) of 34 weeks and favorable clinical conditions are the criteria routinely used for the removal of the feeding tube and the beginning of oral feeding. In the majority of babies, a routine speech therapist evaluation is not done and it is possible that many of these babies, even with 34 weeks PMA, present problems related to their oral sensory-motor system that can contribute to feeding difficulties. Thus, the objective of this study was to determine the motor-oral conditions and the feeding performance in preterm newborns when they were permitted to start oral feeding. 


\section{Patients and Methods}

This was an observational prospective study conducted on preterm newborns admitted to the Neonatal Intensive Care Unit of the University Hospital, Federal University of Santa Maria, RS, Brazil, from May 1 to September 30, 2006. The study was approved by the Research Ethics Committee of UFSM (\#23081016011/2006-58). Newborns with a gestational age of less than 37 weeks at birth were included after their parents and/or persons responsible signed an informed consent form. Exclusion criteria were: presence of congenital head and neck malformations, genetic syndromes, neonatal asphyxia defined by a 5 th min Apgar score of 6 or less, grade III or IV intracranial hemorrhage (4), and kernicterus.

A total of 121 newborns were admitted to the unit during the study period, 74 of them being preterm. Of these, 11 died during the 1 st week, 5 presented neurological involvement, and 15 received their first oral feeding before speech therapist evaluation. Thus, the study sample consisted of 43 newborns with no type of nipple or pacifier being offered before they were started on oral feeding. All were continuously monitored for heart and respiratory rate and oxygen saturation.

At the time of medical prescription of the transition from orogastric tube to oral feeding, the newborns were evaluated by the same experienced speech therapist $10 \mathrm{~min}$ before a feeding. The adaptive oral reflexes of searching, sucking and swallowing, the degree of sucking force, the sucking rhythm, and the presence of sucking-swallowingrespiration coordination were evaluated by non-nutritive sucking using a gloved finger. Force was classified as strong or weak according to the pressure exerted on the finger. The rhythm was considered to be fast when the baby showed approximately 2 sucks/s and a number of sucking bursts equivalent to the time of pause (5). Slow rhythm was considered when the baby did not meet these criteria. Sucking-swallowing-respiration incoordination was considered when a pause occurred in respiration with a decrease in heart rate ( $<100 \mathrm{bpm}$ ) and in oxygen saturation $(<90 \%)$. The nutritional performance of the newborn was evaluated on the basis of competence and overall transfer. The first feeding after speech therapist evaluation was considered for competence, which was calculated by dividing the volume ingested by the time spent in oral feeding $(\mathrm{mL} / \mathrm{min})$. The overall transfer (the percent volume transferred during an entire feeding/total volume order for that feeding) was calculated every day until the day when the infant ingested the entire volume prescribed. The speech therapist evaluation and the first oral feeding were filmed with a JVC camera, model GR-AX25U, and timed with a
Mondaine chronometer for the recording and reconfirmation of the data, which were colleted in real time.

The results were fed into a data bank and analyzed with the STATA10 statistical software. Adjustment tests for data normality were carried out and compared by the Student unpaired $t$-test and $\chi^{2}$ test. A multiple linear regression model that included competence and days for overall transfer of $100 \%$ adjusted for force, rhythm, sucking-swallowing-respiration coordination and adaptive oral reflexes was then used. Data are reported as frequencies, means $\pm S D$, and $P<0.05$ was considered to be statistically significant for univariate analysis and $P<0.10$ for multiple linear regression.

\section{Results}

The study group consisted of 43 preterm infants $(47 \%$ male) with a mean birth weight of $1589 \pm 486 \mathrm{~g}$ (range 790$2800 \mathrm{~g}$ ) and a gestational age of $32.4 \pm 4.3$ weeks (range $28-35$ weeks), $25.6 \%$ were classified as small, $70 \%$ as adequate and $4.4 \%$ as large for gestational age. When they were switched to oral feeding, mean weight was 1817 $\pm 368 \mathrm{~g}$, PMA was $34.6 \pm 1.4$ weeks, and they had $17.6 \pm$ 12 days of life.

The oral motor conditions of the evaluation of nonnutritive sucking and the feeding performance of the preterm newborns are reported in Table 1. Most newborns had strong non-nutritive sucking and a rapid rhythm, although $32.6 \%$ sucked weakly and $23.3 \%$ presented a slow sucking rhythm. Similar results were obtained for the adaptive oral reflexes of searching, sucking and swallowing, with $30 \%$ of the newborns not presenting the three reflexes concomitantly. Coordination of sucking, swallowing and respiration was observed in most of the infants studied (86\%), although $14 \%$ presented incoordination during nonnutritive sucking.

The mean feeding competence of the 43 infants studied was $1.7 \pm 1.3 \mathrm{~mL} / \mathrm{min}$. When evaluated according to sucking force, mean competence was $2.0 \pm 1.4 \mathrm{~mL} / \mathrm{min}$ for infants with strong sucking but was half this value in infants with weak sucking $(P=0.009)$. A similar result was observed for the concomitant occurrence of the adaptive oral reflexes (searching, sucking and swallowing). Competence was greater in the group with the three concomitant reflexes than in the group without their concomitant presence $[1.9 \pm 1.4$ and $1.1 \pm 1.0 \mathrm{~mL} / \mathrm{min}$, respectively $(P=$ $0.032)$.

The feeding performance was also evaluated by overall transfer. Considering a transfer rate of $100 \%$, the infants who were found to have strong sucking, a rapid rhythm, coordination of sucking, swallowing and respiration and 
the concomitant presence of the three adaptive oral reflexes were fully transferred to oral feeding between the 4th and 5th day, while the remaining infants were fully transferred between the 7 th and 11th day $(P<0.05)$.

In a multiple linear regression model, inclusion of the confounding variables (oral motor evaluation) showed a positive association between competence and force (regression coefficient $=0.94 ; \mathrm{SE}=0.42 ; \mathrm{P}=$ 0.03 ), between days for overall transfer of $100 \%$ and rhythm (regression coefficient $=$ 2.14; SE $=1.08 ; \mathrm{P}=0.05$ ) and suckingswallowing-respiration coordination (regression coefficient $=4.44 ; \mathrm{SE}=1.31 ; \mathrm{P}=$ $0.02)$. Thus, the presence of a strong suck implied a better performance for competence. Fast rhythm and presence of suckswallow-breathe coordination decreased the days for an overall transfer of $100 \%$.

\section{Discussion}

The 43 premature infants studied were switched to oral feeding according to the criteria adopted by the Neonatal Unit of the University Hospital of Santa Maria, which recommends the beginning of oral feeding at about the 34th week of PMA, with the infant weighing at least $1500 \mathrm{~g}$ and presenting stable clinical conditions. However, the importance of an evaluation carried out by a skilled professional, such as a speech therapist at the time to start the oral feeding, has not been extensively studied and still is not routinely included in many neonatal intensive care units. Thus, this study sought to show how this evaluation is important to ensure a safe and efficient introduction of oral feeding.

The gestational age is the most studied and used criterion to switch premature infants to oral feeding. It ranges from 32 to 34 weeks in the literature (6), and was also adopted in our study in which the mean age for transition was 34.6 weeks. However, the readiness for oral feeding may depend on other factors in addition to gestational age and a stable clinical condition. Coordination between suck, swallow and respiration is an essential condition to start the oral feeding without risks to the pulmonary and/or the central nervous systems (7). Although the sucking and swallowing functions are already present in preterm infants starting at the 28th week of gestational age they are not effectively coordinated with respiration before the 34 th week (8). In this study, evalua- tion by a speech therapist revealed that $14 \%$ of the preterm infants at the time to start to oral feeding did not present coordination between suck, swallow and respiration and all of them were at PMA of 34 weeks or more. This was also found in a similar study in which only $25 \%$ of the preterm infants showed adequate suck-swallow-respiration coordination at PMA of 34 weeks (9). The oral motor capacity was also evaluated by force, rhythm and the presence of the adaptive oral reflexes. Among the infants studied, $67 \%$ had strong sucking, $77 \%$ had a rapid rhythm and $70 \%$ showed the concomitant presence of the adaptive oral reflexes of searching, sucking and swallowing. The infants who sucked strongly and presented the three adaptive oral reflexes concomitantly showed a significantly greater competence of feeding performance $(2.0$ and $1.9 \mathrm{~mL} / \mathrm{min})$ compared with the remaining infants $(1.1 \mathrm{~mL} / \mathrm{min})$. Although adequate values for competence have been suggested to be the intake of $3 \mathrm{~mL} / \mathrm{min}$ of milk (10), lower values of $1.5 \mathrm{~mL} / \mathrm{min}$ in the first oral feeding were also considered to be indicative of success for preterm newborns with a gestational age of 26 to 29 weeks at birth (11).

The overall transfer was greater in infants with strong sucking, rapid rhythm, the presence of the three oral reflexes and sucking-swallowing-respiration coordination. These infants were completely switched from tube to oral feeding between the 4th and 5th day after the beginning of oral feeding, while the remaining infants were switched 
only between the 7th and 10th day.

Besides the oral motor evaluation, protocols of oral sensory-motor stimulation have been proposed, especially in extremely premature babies, in order to facilitate the transition from tube to oral feeding and thus permit an earlier hospital discharge $(12,13)$. Many of the procedures to which these extremely preterm babies are submitted during hospitalization (tracheal intubation and orogastric tubes) can interfere with oral motor development and consequently with their feeding performance (14). In the present study, the mean gestational age at birth was 32.4 weeks, suggesting that even larger premature babies, who often do not need mechanical ventilation or a tube for

\section{References}

1. Bu'Lock F, Woolridge MW, Baum JD. Development of coordination of sucking, swallowing and breathing: ultrasound study of term and preterm infants. Dev Med Child Neurol 1990; 32: 669-678.

2. Braum MA, Palmer MM. A pilot study of motor-oral dysfunction in "at-risk" infants. Phys Occup Ther Pediatr 1985; 5: 13-25.

3. Gardner SL, Hagedorn MI. Physiologic sequelae of prematurity: the nurse practitioner's role. Part V. Feeding difficulties and growth failure (pathophysiology, cause, and data collection). J Pediatr Health Care 1991; 5: 122-134.

4. Volpe JJ. Intracranial hemorrhage: germinal matrix-intraventricular hemorrhage of the premature infant. In: Volpe JJ (Editor), Neurology of the newborn. 3rd edn. Philadelphia: WB Saunders Company; 1995. p 403-463.

5. Wolff $\mathrm{PH}$. The serial organization of sucking in the young infant. Pediatrics 1968; 42: 943-956.

6. Neiva FCB, Leone CR. Sucking development in preterm newborns and the influence of nonnutritive sucking stimulation. Pediatr Res 2003; 53: 498A.

7. Lau C. Development of oral feeding skills in the preterm infant. Arch Pediatr 2007; 14 (Suppl 1): S35-S41. prolonged periods of time, may have oral sensory-motor difficulties that affect their feeding performance, with the consequent need for intervention.

The data reported here showed that the gestational age criterion associated with a stable clinical condition does not ensure readiness or efficiency to start oral feeding in preterm infants. This study showed that their feeding performance depended on a favorable oral motor condition, more precisely, on strong sucking, rapid rhythm and on sucking-swallowing-respiration coordination. For this reason, evaluation by a speech therapist is necessary to determine the beginning of oral feeding with safety.

8. Lau C, Schanler RJ. Oral motor function in the neonate. Clin Perinatol 1996; 23: 161-178.

9. Mizuno K, Ueda A. The maturation and coordination of sucking, swallowing, and respiration in preterm infants. $J$ Pediatr 2003; 142: 36-40.

10. Lau C, Smith EO, Schanler RJ. Coordination of suck-swallow and swallow respiration in preterm infants. Acta Paediatr 2003; 92: 721-727.

11. Lau C, Sheena HR, Shulman RJ, Schanler RJ. Oral feeding in low birth weight infants. J Pediatr 1997; 130: 561-569.

12. Fucile $\mathrm{S}$, Gisel E, Lau C. Oral stimulation accelerates the transition from tube to oral feeding in preterm infants. $J$ Pediatr 2002; 141: 230-236.

13. Rocha AD, Moreira ME, Pimenta HP, Ramos JR, Lucena $\mathrm{SL}$. A randomized study of the efficacy of sensory-motororal stimulation and non-nutritive sucking in very low birthweight infant. Early Hum Dev 2007; 83: 385-388.

14. Bier JA, Ferguson A, Cho C, Oh W, Vohr BR. The oral motor development of low-birth-weight infants who underwent orotracheal intubation during the neonatal period. Am J Dis Child 1993; 147: 858-862. 